\title{
LIXIVIAÇÃO SOB PRESSÃO DE ÓXIDO WAELZ COM CARBONATO DE SÓDIO PARA REMOÇÃO DE HALOGÊNIOS*
}

\author{
Júlia Mont'Alverne Martins ${ }^{1}$ \\ Antonio Rimaci Miguel $\mathrm{Jr}^{2}$ \\ Fabiana Maria Teixeira ${ }^{3}$ \\ Pablo dos Santos Pina ${ }^{4}$ \\ Lucas de Carvalho Cesar ${ }^{5}$ \\ Luiz Flávio da Silva ${ }^{6}$ \\ Francisco Cezar Freire ${ }^{7}$
}

\section{Resumo}

A Votorantim Metais processa pós de aciaria elétrica (PAE) e outros resíduos contendo zinco em sua unidade de Juiz de Fora, por meio do processo Waelz. Devido ao alto teor de óxido de cálcio $(\mathrm{CaO})$ nos resíduos processados, a etapa de desalogenação apresenta baixo rendimento de remoção de fluoretos -aproximadamente $20 \%$. O processo de lixiviação sob pressão foi testado para o tratamento de amostras de óxido Waelz (O.W.) da Votorantim Metais com o objetivo de lixiviar os cloretos e fluoretos contidos nesse material. Os parâmetros avaliados foram: temperatura de lixiviação $\left(120^{\circ} \mathrm{C}\right.$ a $\left.180^{\circ} \mathrm{C}\right) \mathrm{e}$ razão de carbonato de sódio $\left(\mathrm{Na}_{2} \mathrm{CO}_{3}\right)$ adicionado $(100 \mathrm{~kg} / \mathrm{t}$ a $200 \mathrm{~kg} / \mathrm{t}$ óxido Waelz), em um reator piloto com capacidade de 10L. A técnica mostrou-se eficiente para lixiviação de cloretos, com remoção acima de $94,2 \%$. Os resultados para fluoretos obtidos apresentaram variação entre $63,0 \%$ e $96,2 \%$ de rendimento de lixiviação e uma forte correlação dos resultados com a temperatura. Esse processo mostrou-se eficiente em escala de bancada, apresentando rendimento de extração superior a $90 \%$ para a remoção de halogênios em temperaturas acima de $150^{\circ} \mathrm{C}$ e adição de $\mathrm{Na}_{2} \mathrm{CO}_{3}$ de $140 \mathrm{~kg} / \mathrm{t} \mathrm{O}$.W.

Palavras-chave: Reciclagem; Lixiviação sob pressão; Óxido Waelz; Desalogenação.

\section{WAELZ OXIDE PRESSURE LEACHING WITH SODA ASH FOR HALOGENS REMOVAL Abstract}

Votorantim Metais processes electric arc furnace (EAF) dust and other zinc bearing materials throught the Waelz process at its Juiz de Fora unit. Due to high calcium oxide $(\mathrm{CaO})$ content in the residues feed of the plant, the dehalogenation stage has low efficience on fluorine removal, of about $20 \%$. The application of pressure leaching process in the treatment of Waelz oxide of Votorantim Metais was studied aiming leach and wash out chlorine and fluorine of the oxide matriz. The parameters evalueted were leaching temperature $\left(120\right.$ to $\left.180^{\circ} \mathrm{C}\right)$ and addition ratio of sodium carbonate (100 to $200 \mathrm{~kg} / \mathrm{t}$ Waelz oxide), in a pilot scale reactor. The technique was efficiente to leach chlorine compounds, the minimum chloride removal yield was $94,2 \%$. The fluorine compounds removal yield ranged from $63,0 \%$ to $96,2 \%$ with strong correlation of the results with the temperature of the leaching tests. The proposed process show good halogens leaching yield, in pilot scale, with results superior to $90 \%$ in temperature over $150^{\circ} \mathrm{C}$ and addition ratio of $\mathrm{Na}_{2} \mathrm{CO}_{3}$ over $140 \mathrm{~kg} / \mathrm{t}$ Waelz oxide.

Keywords: Zinc recicling; Pressure leaching; Waelz oxide; Dehalogenation.

1 Engenheira química, B. $^{\text {ela }}$, Engenheira de Tecnologia, Departamento de Tecnologia, Votorantim Metais Zinco S.A., Juiz de Fora, Minas Gerais, Brasil.

2 Engenheiro químico, B. el, Consultor, Área de Desenvolvimento de Produtos e Processos Industriais, SENAI CIMATEC DR/BA, Bahia, Brasil.

3 Engenheira de materiais, B. $^{\text {ela }}$, Engenheira de Tecnologia, Departamento de Tecnologia, Votorantim Metais Zinco S.A., Juiz de Fora, Minas Gerais, Brasil.

4 Engenheiro metalurgista, $M^{e}$, Gerente de Tecnologia, Departamento de Tecnologia, Votorantim Metais Zinco S.A., Juiz de Fora, Minas Gerais, Brasil.

5 Engenheiro químico, $B .^{e l}$, Consultor, Área de Desenvolvimento de Produtos e Processos Industriais, SENAI CIMATEC DR/BA, Bahia, Brasil.

6 Engenheiro Mecânico, $B .^{\text {el }}$, consultor II, Área de Desenvolvimento de Produtos e Processos Industriais, SENAI CIMATEC DR/BA, Bahia, Brasil.

7 Engenheiro Químico, $M^{e}$, Gerente da área de Tecnologia em processos, Área de Desenvolvimento de Produtos e Processos Industriais, SENAI CIMATEC DR/BA, Bahia, Brasil. 


\section{INTRODUÇÃO}

Em dezembro de 2011, a Votorantim Metais iniciou a operação de uma nova planta para processamento de pós de aciaria elétrica (PAE) e outros resíduos contendo zinco em sua unidade de Juiz de Fora. Esse tratamento é realizado em um forno Waelz e o óxido Waelz gerado é lixiviado com solução de carbonato de sódio para remoção de halogênios $(\mathrm{Cl}$ e $\mathrm{F})$ em reatores abertos a $80^{\circ} \mathrm{C}$, etapa denominada desalogenação. Atualmente, o tratamento de matérias primas secundárias corresponde a cerca de $21 \%$ de todo o zinco produzido na unidade.

Devido ao alto teor de óxido de cálcio $(\mathrm{CaO})$ nos pós de aciaria elétrica processados na unidade, o teor de $\mathrm{CaO}$ no óxido Waelz também encontra-se acima do limite esperado, atualmente em torno de $1,0 \%$. Observou-se que quanto maior o teor de $\mathrm{CaO}$ no óxido Waelz, menor é a extração de flúor na desalogenação, devido à presença de $\mathrm{CaF}_{2}$, o qual é de difícil decomposição, mesmo com a adição de carbonato de sódio, limitando a eficiência de remoção de flúor (KOMORI, 2015) [1]. As premissas do projeto eram de gerar um óxido com teor de $\mathrm{CaO}$ de $0,5 \%$ e com isso a expectativa era obter cerca de $80 \%$ de rendimento de extração de flúor na etapa de desalogenação. No entanto, o nível de remoção de fluoretos observado atualmente é da ordem de $20 \%$.

A unidade de Juiz de Fora realiza o processamento de matérias primas primárias e secundárias para produção de zinco metálico por meio da rota de processo: ustulação, lixiviação e eletrólise, mais conhecida como Roasting - Leaching - Electrowinnig (RLE). Cloro e flúor são prejudiciais na etapa de eletrólise do zinco, onde é realizada a redução eletrolítica do metal de interesse em uma solução de sulfato de zinco purificada.

Com o objetivo de adequar o teor de fluoretos para a eletrólise, uma segunda etapa de desalogenação do óxido Waelz foi implementada, através da passagem desse material no forno ustulador. Os níveis de remoção de fluoretos e cloretos na primeira etapa hidrometalúrgica são de aproximadamente $20 \%$ e $90 \%$, respectivamente, e os níveis acumulados após o ustulador chegam a $95 \%$ para fluoretos e $99 \%$ para cloretos. Entretanto, a necessidade da segunda etapa de desalogenação em forno ustulador tem representado uma restrição para a expansão da capacidade de tratamento de PAE, uma vez que a capacidade de processamento de óxido Waelz no ustulador é limitada.

A princípio, existem duas formas de realizar a desalogenação do óxido Waelz: o tratamento térmico a temperaturas elevadas, o qual realiza a vaporização dos sais halogenados e o tratamento hidrometalúrgico que utiliza o princípio de solubilização dos sais em soluções aquosas (RUETTEN, 2010) [2]. Várias unidades que empregam o processo Waelz no mundo operam etapas de tratamento do óxido Waelz crú para remoção de halogênios similares à utilizada em Juiz de Fora, realizando a dissolução de $\mathrm{Cl}$ e $\mathrm{F}$ através da lixiviação com $\mathrm{Na}_{2} \mathrm{CO}_{3}$ em temperaturas entre $50^{\circ} \mathrm{C}$ e $70^{\circ} \mathrm{C}$. Entre essas plantas estão as unidades da Befesa Zinc S.L.U., porém elas não apresentam problemas relacionados à remoção de fluoretos, devido às características dos resíduos tratados (GAMROTH, 2010) [3].

Diversos outros processos foram testados ou são utilizados, como a purificação prévia à entrada no forno Waelz dos pós de aciaria, principalmente para remoção de cloro e chumbo (KRUPKA, 2010) [4]. A empresa Shisaka Smelting Co., LTD desenvolveu um processo para a remoção de flúor no forno Waelz com adição de $\mathrm{CaO}$ para aumentar a fixação do elemento na escória e reduzir os teores no óxido Waelz, pois apresentava o mesmo problema para remoção de flúor na desalogenação (KOMORI, 2015) [1]. 
Segundo Ruetten (2010) [2], processos hidrometalúrgicos para a dissolução de sais de cloreto e fluoreto com a adição de $\mathrm{Na}_{2} \mathrm{CO}_{3}$ são mais eficientes com o aumento da temperatura do sistema para dissolução de alguns compostos, especialmente o $\mathrm{CaF}_{2}$. Para tal, a utilização de uma autoclave para realização da lixiviação em temperaturas mais elevadas é uma potencial tecnologia.

Espera-se que os parâmetros de processo da autoclave favoreçam a reação entre carbonato de sódio e os fluoretos e cloretos contidos no óxido, gerando fluoreto e cloreto de sódio (conforme reações abaixo), que são sais solúveis e que podem ser eliminados no filtrado, permitindo a obtenção de teores de halogênios na torta dentro das especificações do processo, visando atender à eletrólise.

(1) $\mathrm{PbCl}_{2}+\mathrm{Na}_{2} \mathrm{CO}_{3} \leftrightarrow \mathrm{PbCO}_{3}+2 \mathrm{NaCl}$

(2) $2 \mathrm{~Pb}(\mathrm{OH}) \mathrm{Cl}+\mathrm{Na}_{2} \mathrm{CO}_{3} \leftrightarrow \mathrm{Pb}(\mathrm{OH})_{2}+\mathrm{PbCO}_{3}+2 \mathrm{NaCl}$

(3) $2 \mathrm{Zn}(\mathrm{OH}) \mathrm{Cl}+\mathrm{Na}_{2} \mathrm{CO}_{3} \leftrightarrow \mathrm{Zn}(\mathrm{OH})_{2}+\mathrm{ZnCO}_{3}+2 \mathrm{NaCl}$

(4) $\mathrm{Na}_{2} \mathrm{CO}_{3}+\mathrm{CaF}_{2} \leftrightarrow \mathrm{CaCO}_{3}+2 \mathrm{NaF}$

Tendo em vista os fatos apresentados e os aspectos operacionais da unidade de Juiz de Fora da Votorantim Metais, o objetivo desse trabalho, desenvolvido em parceria com o SENAI CIMATEC DR/BA, é avaliar a eficiência de remoção de halogênios presentes no óxido Waelz, com foco na remoção de flúor, por meio de lixiviação sob pressão do óxido Waelz.

\section{MATERIAIS E MÉTODOS}

Foram avaliados dois parâmetros na lixiviação sob pressão do óxido Waelz: a temperatura de lixiviação $\left(120^{\circ} \mathrm{C}\right.$ a $\left.180^{\circ} \mathrm{C}\right)$ e a razão de carbonato de sódio $\left(\mathrm{Na}_{2} \mathrm{CO}_{3}\right)$ adicionado (100kg/t a $200 \mathrm{~kg} / \mathrm{t}$ de óxido Waelz). O tempo de residência foi fixado em 90 minutos e o percentual de sólidos em $25 \%$ (p/p), sob agitação constante a 250rpm. O planejamento dos experimentos foi elaborado com três níveis e dois fatores em duplicata, para verificar a influência da temperatura e da dosagem de carbonato na remoção de fluoretos e cloretos através da ferramenta Design of Experiments (DOE) do software Minitab 17. As amostras foram consideradas como blocos na avaliação estatística para verificar a influência de diferentes matrizes na remoção de halogênios e a ordem dos experimentos foi aleatorizada.

O equipamento utilizado foi uma autoclave da marca PARR, modelo 4552-T-SS-230VS-2000-4843 com capacidade de 10L, pressão de operação de 2-20bar e temperatura de operação $120-200^{\circ} \mathrm{C}$. Os testes foram conduzidos em regime de batelada.

\section{1 Óxido Waelz}

Foram utilizadas duas amostras de óxido Waelz geradas na unidade de Juiz de Fora da Votorantim Metais em períodos distintos de operação do forno Waelz. A composição química de cada amostra é apresentada na Tabela 1. 
Tabela 1. Análise química do óxido Waelz utilizado

\begin{tabular}{lrr}
\multicolumn{1}{c}{ Óxido Waelz } & Amostra 1 & Amostra 2 \\
\hline $\mathbf{F ~ ( \% ) ~}$ & $0,4994 \%$ & $0,5370 \%$ \\
\hline $\mathbf{C l}(\%)$ & $8,04 \%$ & $8,66 \%$ \\
\hline $\mathbf{Z n ~ ( \% )}$ & $56,67 \%$ & $52,31 \%$ \\
\hline $\mathbf{P b}(\%)$ & $4,46 \%$ & $5,38 \%$ \\
\hline Fe (\%) & $3,03 \%$ & $2,60 \%$ \\
\hline $\mathrm{CaO}(\%)$ & $1,02 \%$ & $0,82 \%$ \\
\hline
\end{tabular}

\subsection{Procedimento de lixiviação sob pressão em autoclave}

O procedimento para lixiviação sob pressão consiste em repolpar o óxido Waelz halogenado com água, sob agitação vigorosa, em chapa elétrica até a temperatura de $60^{\circ} \mathrm{C}$ e adicionar o carbonato de sódio. Foram utilizados $1.000 \mathrm{~g}$ de óxido Waelz em cada teste e a adição de água e de $\mathrm{Na}_{2} \mathrm{CO}_{3}$ foi calculada a partir desse valor, de forma que a massa total final da polpa na entrada tivesse sempre $4.000 \mathrm{~g}$ de massa. Então essa polpa é transferida para o reator piloto e são feitos os ajustes dos parâmetros em avaliação, o teste inicia-se com o atingimento da temperatura selecionada. A amostra então é filtrada e alíquotas da solução e da torta são coletadas para análise. Neste trabalho não foram realizadas etapas de lavagem da polpa de óxido Waelz desalogenado, como é previsto no processo industrial. O volume da solução, a massa e a umidade da torta são medidos após a conclusão dos ensaios.

\section{RESULTADOS E DISCUSSÃO}

Os resultados dos ensaios de lixiviação sob pressão do óxido Waelz estão apresentados na Tabela 1. Para o cloro todos os ensaios apresentaram resultados de rendimento de extração superiores a $94,2 \%$. Para a extração de flúor, os resultados variaram entre $63,0 \%$ e $96,2 \%$.

Tabela 2. Parâmetros e resultados dos ensaios de lixiviação sob pressão

\begin{tabular}{ccccccc}
$\mathbf{n}^{\circ}$ Ensaio & Amostra & $\begin{array}{c}\text { Temperatura } \\
\left({ }^{\circ} \mathbf{C}\right)\end{array}$ & $\begin{array}{c}\text { Pressão } \\
\text { Máx. (PSI) }\end{array}$ & $\begin{array}{c}\mathbf{N a}_{2} \mathbf{C O}_{3} \\
(\mathbf{k g} / \mathbf{t ~ d e ~ O . W . )}\end{array}$ & $\begin{array}{c}\text { Rendimento } \\
\text { Ext. } \mathbf{F ~ ( \% ) ~}\end{array}$ & $\begin{array}{c}\text { Rendimento } \\
\text { Ext. } \mathbf{C l}(\%)\end{array}$ \\
\hline 1 & 1 & 180 & 158 & 200 & $92,0 \%$ & $94,2 \%$ \\
\hline 2 & 1 & 180 & 153 & 150 & $94,1 \%$ & $95,4 \%$ \\
\hline 3 & 1 & 120 & 39 & 200 & $79,3 \%$ & $95,6 \%$ \\
\hline 4 & 1 & 150 & 74 & 200 & $95,9 \%$ & $97,7 \%$ \\
\hline 5 & 1 & 120 & 35 & 100 & $73,6 \%$ & $96,5 \%$ \\
\hline 6 & 1 & 150 & 70 & 150 & $91,7 \%$ & $96,0 \%$ \\
\hline 7 & 1 & 120 & 39 & 150 & $74,6 \%$ & $97,2 \%$ \\
\hline 8 & 1 & 180 & 145 & 100 & $96,2 \%$ & $98,2 \%$ \\
\hline 9 & 1 & 150 & 76 & 100 & $88,9 \%$ & $97,9 \%$ \\
\hline 10 & 2 & 150 & 76 & 100 & $82,2 \%$ & $96,9 \%$ \\
\hline 11 & 2 & 150 & 77 & 150 & $89,7 \%$ & $96,3 \%$ \\
\hline 12 & 2 & 180 & 142 & 100 & $95,9 \%$ & $98,4 \%$ \\
\hline 13 & 2 & 120 & 40 & 100 & $63,0 \%$ & $98,4 \%$ \\
\hline 14 & 2 & 180 & 146 & 200 & $95,9 \%$ & $97,5 \%$ \\
\hline
\end{tabular}




\begin{tabular}{ccccccc}
$71^{\circ}$ Congresso Anval & & & & & \multicolumn{2}{c}{$\begin{array}{c}\text { abm } \\
\text { ibek } \\
\text { ISN 1516-392X }\end{array}$} \\
\hline 15 & 2 & 120 & 37 & 200 & $72,2 \%$ & $95,1 \%$ \\
\hline 16 & 2 & 120 & 33 & 150 & $66,4 \%$ & $96,6 \%$ \\
\hline 17 & 2 & 180 & 144 & 150 & $95,3 \%$ & $96,3 \%$ \\
\hline 18 & 2 & 150 & 71 & 200 & $83,7 \%$ & $98,1 \%$ \\
\hline
\end{tabular}

\subsection{Efeito da temperatura}

Como é possível observar na Figura 1, quanto maior a temperatura de realização da lixiviação, maiores os rendimentos de extração de flúor. Os ensaios realizados à temperatura de $120^{\circ} \mathrm{C}$ obtiveram rendimento médio de $71,5 \%$. Nos ensaios à $150^{\circ} \mathrm{C}$, essa média aumentou para $88,7 \%$ e na temperatura mais elevada avaliada, $180^{\circ} \mathrm{C}$, a média dos ensaios apresentou rendimento de $94,9 \%$.

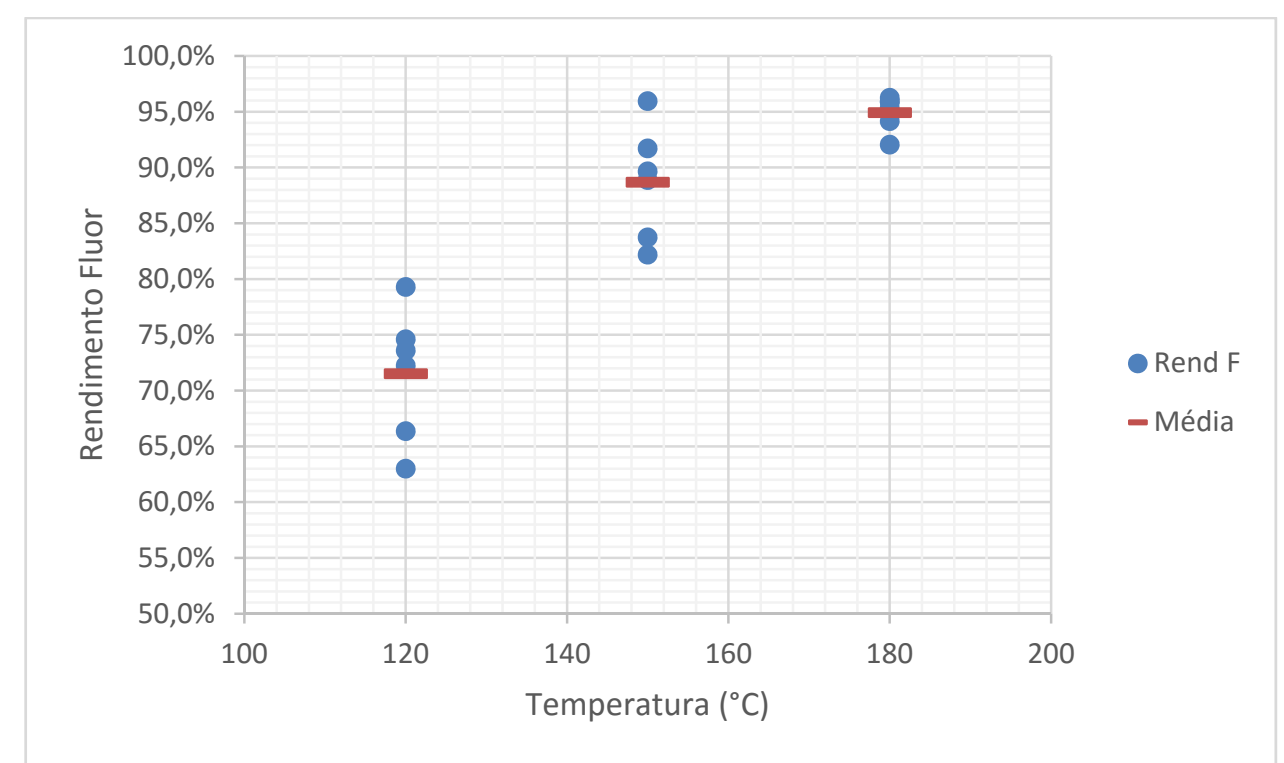

Figura 1. Gráfico de rendimento de extração de flúor por temperatura.

Esses resultados evidenciam que há influência direta da temperatura no rendimento de extração de fluoretos através da formação de sais solúveis desse elemento.

\subsection{Efeito da adição de carbonato de sódio}

Os resultados dos ensaios, avaliados sob o ponto de vista de adição de carbonato de sódio, não apresentaram correlação significativa desse parâmetro na extração de flúor, como é possível observar pela dispersão dos dados da Figura 2. 


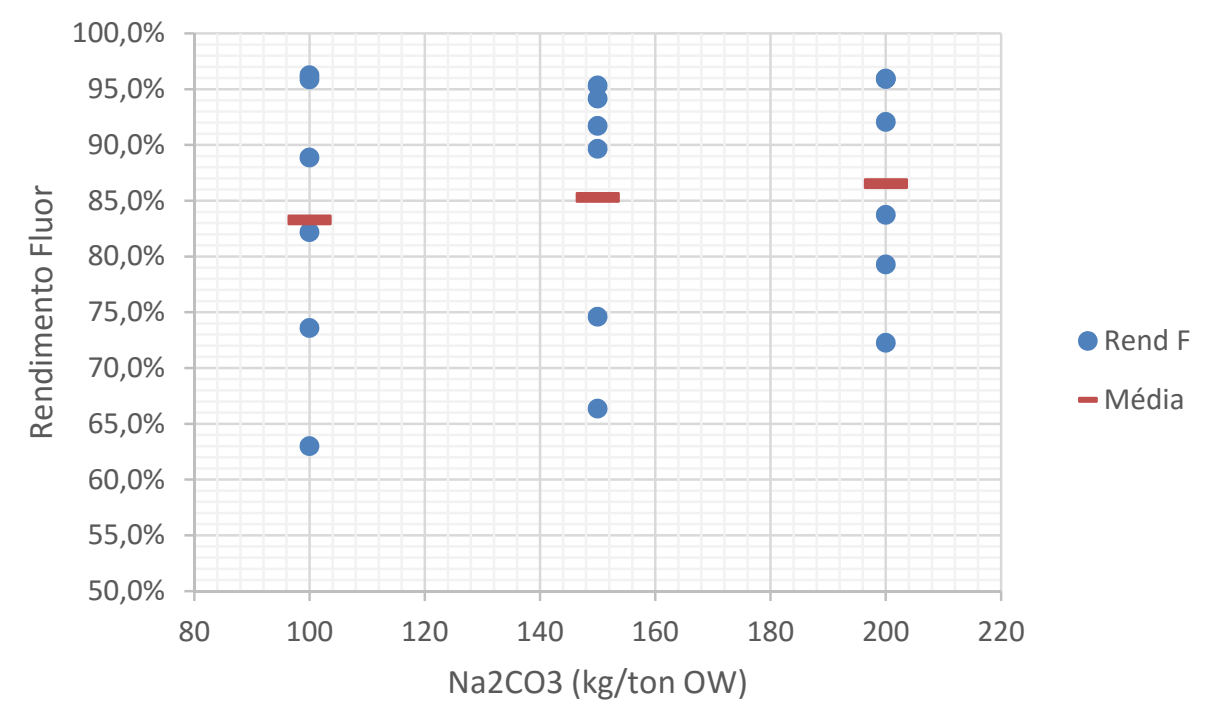

Figura 2. Gráfico de rendimento de extração de flúor por adição de carbonato de sódio.

As médias de rendimento de extração de flúor para adição de carbonato de 100, 150 e $200 \mathrm{~kg} / \mathrm{t} \mathrm{O.W}$. foram, respectivamente, $83,3 \%, 85,3 \%$ e $86,5 \%$. A diferença entre o menor e o maior rendimento foi de apenas 3,2\%, enquanto a avaliação sob a perspectiva da temperatura teve amplitude de $23,4 \%$.

No gráfico de contorno elaborado através do software Minitab 17 (Figura 3), que correlaciona os dados de todos os testes realizados, verifica-se que trabalhando com adição de carbonato de sódio de $140 \mathrm{~kg} / \mathrm{t}$ O.W. e temperatura acima de $150^{\circ} \mathrm{C}$, obtemse rendimentos superiores a $90 \%$ de extração de flúor para todas as configurações possíveis.

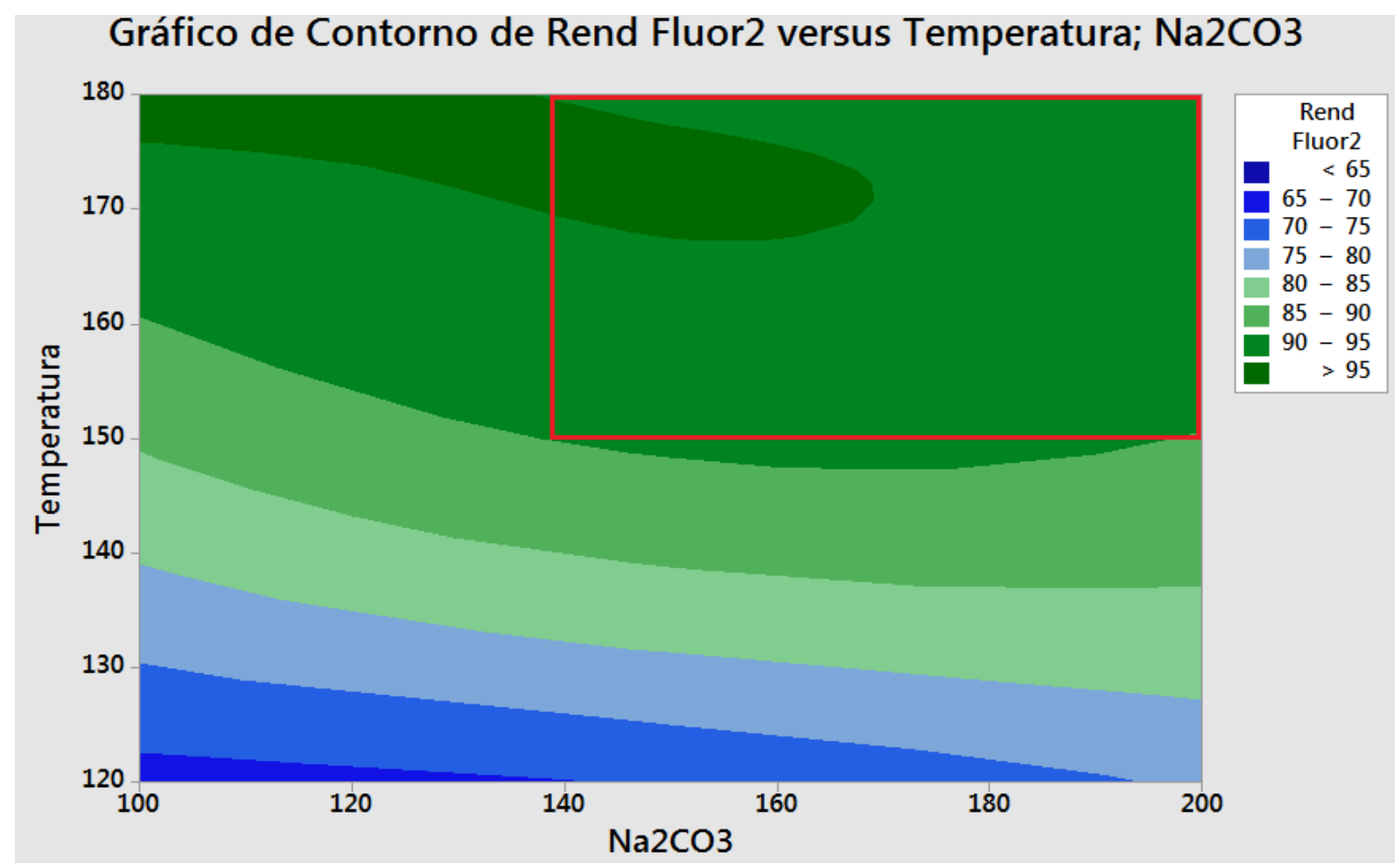

Figura 3. Gráfico de contorno para os dados dos ensaios de lixiviação sob pressão. 
A análise estatística dos testes foi realizada através da ferramenta de Regressão Fatorial Geral do software Minitab 17 (Figura 4). Além dos fatores temperatura e carbonato de sódio $\left(\mathrm{Na}_{2} \mathrm{CO}_{3}\right)$, as amostras foram consideradas como blocos para avaliar se havia alguma influência na composição de cada amostra nos resultados dos testes. Ao nível de significância de $95 \%$, a regresão indica que a temperatura e os blocos são fatores significativos (Pvalor $<0,05)$ e o carbonato de sódio é rejeitado (Pvalor $>0,05)$. Isso confirma a avaliação realizada visualmente por meio dos gráficos. Os blocos são significativos, indicando que a matriz da amostra é importante para os resultados dos testes. Considerando que as amostras apresentavam composição química similar, é possível deduzir que variações grandes nas matrizes das amostras podem impactar nos resultados de desalogenação.

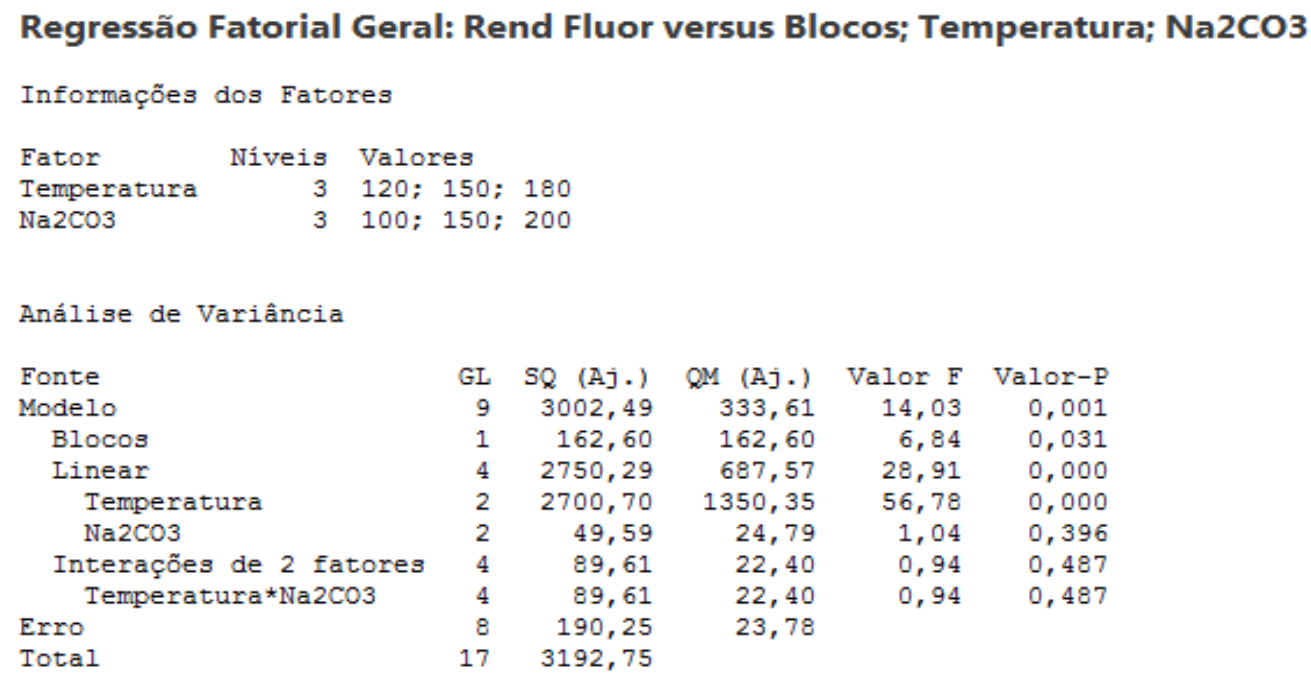

Figura 4: Regressão dos dados de teste - autoclave.

Plantas de desalogenação comumente operam etapas de lavagem do óxido Waelz desalogenado para remoção da solução contendo sais de cloreto e fluoreto que ficam na umidade da torta filtrada. Essa etapa não foi realizada neste estudo, portanto, espera-se que com a inclusão de etapas de lavagem, os resultados de remoção de halogênios apresentem rendimentos superiores aos reportados nesta pesquisa.

\section{CONCLUSÃO}

A técnica de lixiviação sob pressão do óxido Waelz produzido na Votorantim Metais, com adição de carbonato de sódio, foi desenvolvida para remoção de halogênios por meio da formação de sais solúveis. Essa técnica se mostrou eficiente para lixiviação de cloretos, com resultados acima de $94,2 \%$ de rendimento. Os resultados para fluoretos apresentaram variação entre $63,0 \%$ e $96,2 \%$ de rendimento de lixiviação e uma forte correlação dos resultados com a temperatura de realização dos testes. Esses resultados são muito superiores aos valores obtidos através da lixiviação em reatores abertos, da ordem de $20 \%$, atualmente em operação na unidade industrial da Votorantim Metais.

Verificou-se que são obtidos rendimentos de extração superiores a $90 \%$, para a remoção de halogênios (cloretos e fluoretos) em temperaturas acima de $150^{\circ} \mathrm{C}$ e adição de $\mathrm{Na}_{2} \mathrm{CO}_{3}$ a partir de $140 \mathrm{~kg} / \mathrm{t} \mathrm{O}$.W. Além da temperatura, há influência da composição da amostra nos resultados de remoção de halogênios, sendo o teor de $\mathrm{CaO}$ um parâmetro já identificado como influente nesse processo. 


\section{Agradecimentos}

Os autores agradecem à empresa Votorantim Metais e ao SENAI CIMATEC DR/BA que desenvolveram o projeto Remoção de Cloretos e Fluoretos de Óxido Waelz, incentivado pelo Ministério da Ciência Tecnologia e Inovação através da EMBRAPII (Empresa Brasileira de Pesquisa e Inovação Industrial).

\section{REFERÊNCIAS}

1 Komori S, Fujiyama H, Watanabe H, Tomoda K. Recovering crude zinc oxide from EAF dust at Shisaka Smelting Co., LTD. PbZn GDMB. 2015: 831-841.

2 Ruetten J. Different ways of using Waelz ozide - Overview and Evaluation. PbZn GDMB. 2010: 841-849.

3 Gamroth M, Mager K. SDHL Waelz Technology: State os the Art for Recycling of zinccontaining residues. PbZn GDMB. 2010: 861-870.

4 Krupka D, Kapias P, Czekaj J, Galicki J, Jakubowski J. Modernization of EAF cust Processing Technology at Boleslaw Recicling Ltd., Poland. PbZn GDMB. 2010: 883888.

5 Takayama T, Magalhães W, Santos FM. Treatment of Secondary Raw Materials at Juiz de Fora zinc Smelter in Brazil. PbZn GDMB. 2015: 931-951. 